\title{
Summative Evaluation of Physician-Pharmacist Collaboration Management
}

Rachel J. Finkelstein ${ }^{1}$, Natalie D. Blaine ${ }^{1}$, Christopher P. Parker ${ }^{1}$, Mark W. Vander Weg ${ }^{2-4}$, Barry L. Carter ${ }^{1,5}$

${ }^{1}$ Department of Pharmacy Practice \& Science, University of lowa College of Pharmacy, lowa City, lowa, United States

${ }^{2}$ Department of Internal Medicine, Carver College of Medicine, lowa City, lowa, United States

${ }^{3}$ The lowa City Veterans Administration Health Care System, lowa City, lowa, United States

${ }^{4}$ Department of Psychological and Brain Sciences, University of lowa College of Liberal Arts and Sciences, lowa City, lowa, United States

${ }^{5}$ Department of Family Medicine, University of lowa Roy J and Lucille A. Carver College of Medicine, lowa City, lowa, United States

*Corresponding author: Rachel J. Finkelstein, 115 S. Grand Ave, Room S533, College of Pharmacy, University of lowa, lowa City, IA, 52242, United States, Tel: 319-335-6527; Fax: 319-384-1728; E-mail: rachel-finkelstein@uiowa.edu

Received: December 31, 2015, Accepted: February29, 2016, Published: March 10, 2016

Copyright: (c) 2016 Finkelstein RJ, et al. This is an open-access article distributed under the terms of the Creative Commons Attribution License, which permits unrestricted use, distribution and reproduction in any medium, provided the original author and source are credited.

\section{Abstract}

Background: Studies have shown physician-pharmacist collaboration can improve outcomes and management of chronic disease states; however, there is limited knowledge of the barriers to implementing these models.

Objective: To evaluate the perceived effectiveness of and barriers to physician-pharmacist collaborative management (PPCM) in diverse primary care medical offices.

Methods: Prospective cluster-randomized mixed methods trial in 32 primary care offices. Each medical office was randomized to a 9 or 24 month hypertension intervention or a 9 month asthma intervention with usual care hypertension management. A post study summative evaluation of physician and pharmacists was conducted to identify obstacles and suggest facilitators for implementation of PPCM in primary care offices.

Results: A total of 63 physicians and 26 pharmacists from 27 (84\%) and $26(81 \%)$ offices, respectively, completed the summative evaluation. Collaboration for the duration of the Collaboration Among Pharmacists and Physicians To Improve Outcomes Now (CAPTION) Trial resulted in expanded clinical pharmacy services for the management of chronic diseases. Both physicians and pharmacists participating in the study reported that physicians at their sites were generally receptive to pharmacist's recommendations both prior to and following the study. Physicians appear to be confident in the expertise of pharmacists and their ability to manage chronic diseases. Over $98 \%$ of physicians reported accepting pharmacist recommendations. In addition, $100 \%$ of responding physicians and pharmacists believed the model implemented in the CAPTION Trial could be expanded to disease states beyond blood pressure (BP) and asthma. Collaboration reportedly increased as physician workload became heavier. A major reported barrier to collaboration was access to pharmacists including conflicts with other non-clinical responsibilities. Collaboration had a positive impact on patient-provider relationships and $100 \%$ of the surveyed physicians responded that they believe a pharmacist's involvement in chronic disease management improved patient outcomes. When asked if pharmacists should be recognized as healthcare providers, $95 \%$ of physicians responded affirmatively.

Conclusions: The results of this summative evaluation indicate both physicians and pharmacists perceived collaboration in primary care settings as a means to improve the quality of patient care and treatment outcomes. Physicians and pharmacists perceived collaborative care as well received by patients and that it strengthened patient-provider relationships. Physicians' desire to collaborate with pharmacists to manage chronic diseases is promising but will require ongoing efforts to develop and maintain positive professional relationships as well as reimbursement strategies for clinical services provided by pharmacists.

Keywords: Cardiovascular disease; Pharmacist management; Clinical trial; Health care teams; Physician/ pharmacist relationship; Coordinated care; Pharmacist

\section{Introduction}

As the health care system adapts to the growing population living with chronic disease there has been a shift in the delivery of primary care towards a team-based, patient-centered model $[1,2]$. The benefits of including a pharmacist as a member of a collaborative medical team have been well established. Several systematic reviews and meta- analyses have shown the positive impact pharmacists have on improving BP control and managing other cardiovascular risk factors [3-5].

The American College of Clinical Pharmacy (ACCP) Ambulatory Care Practice and Research Network (PRN) recently published a paper stressing the importance of pharmacists in the optimization of 
medication management in the Patient Centered Medical Home (PCMH) and identify pharmacists as an essential component to the success of the PCMH model. Pharmacy services highlighted in the PRN paper include: conducting comprehensive medical reviews to identify, prevent and resolve medication-related problems, optimizing medication regimens to achieve treatment goals, recommending costeffective therapies, and addressing adherence [6].

PPCM is a team-based care model, consistent with the medical home, by which pharmacists work directly with patients' primary care providers (PCP) to optimize medication therapy and provide patient education. Under the physician-pharmacist collaborative management (PPCM) model, the physician-pharmacist relationship allows the pharmacist to assume responsibilities as delegated by the PCP. One of us (BLC) has extensively evaluated this model to improve BP control in several studies [7-13].

There have been other studies that have evaluated the barriers to and physician perceptions of integrating pharmacists into team models $[14,15]$ including a recently published case study by Snyder et al which examined 3 community-based sites utilizing provider-pharmacist collaborative drug therapy management [16]. To our knowledge none have evaluated barriers and facilitators in a large, randomized, multihealth system clinical trial. The results from both the BP and asthma interventions from the CAPTION Trial have recently been published $[17,18]$. Not only was the intervention shown to improve blood pressure control, it was also shown to be cost effective considering both cost of medications and pharmacist and physician time [19]. This study included a mixed-methods approach including surveys and interviews of physicians and intervention pharmacists in order to evaluate barriers and facilitators to PPCM. The objective of the present summative evaluation was to evaluate the perceived effectiveness of and barriers to the PPCM model from the CAPTION trial.

\section{Methods}

The background and study design of the CAPTION Trial was previously reported [18]. The study was a 5-year, prospective, clusterrandomized multi-center clinical trial in 32 medical offices in 15 states throughout the United States. The goal was to evaluate whether a collaborative model between physicians and pharmacists could improve and sustain BP in diverse primary care offices with high minority populations. Additionally, the study evaluated if BP control deteriorates after discontinuation of the 9-month intervention compared to continuing the intervention for 24 months. Offices were randomized to either a 9 month BP intervention, a 24 month BP intervention or usual care. The pharmacists in usual care offices provided an asthma intervention. An evaluation conducted prior to the study found that all medical offices employed clinical pharmacists on staff or faculty with a mean of 1.9 pharmacists per office [20]. The pharmacists spent an average of 2.1 days per week directly in patient care and offices were covered by a pharmacist an average of $82 \%$ of the time. Most offices have had clinical pharmacy services for multiple years, with $83 \%$ having a clinical pharmacist for over 5 years [20].

\section{Qualitative research design}

After completion of the PPCM intervention, emails were sent to 26 pharmacists and 443 physicians at the 32 study sites inviting them to participate in the summative evaluation with a goal of interviewing 3 physicians from each site. A study investigator then contacted both groups via telephone to gain insight on the strengths, weaknesses, perceived effectiveness, and future direction of the study PPCM intervention as well as suggestions for better integrating PPCM into the structure of usual primary care. The interviewer was a licensed pharmacist, who was not involved previous work or intervention in CAPTION nor had previous interactions with the physicians or pharmacists being interviewed. He was trained by the primary investigator (BC) prior to conducting the interviews. The study was approved by the University of Iowa Institutional Review Board (IRB) and by the local IRB for each study site.

The semi-structured 10-15 minutes, one-on-one telephone interviews were scripted to assure consistent questioning. Notes from the interviews were transcribed and reviewed to ensure that important issues were correctly captured. Consent was assumed when the telephone respondent agreed to participate in the interview.

The evaluation was based on a summative evaluation used previously by the CAPTION investigator [13] and was designed to capture the observations of physicians and pharmacists on type and frequency of recommendations approved, attitudes of physicians regarding pharmacists' role in care, facilitators and barriers to incorporating pharmacists into primary care offices, and suggestions for improving the existing arrangements (Table 1).

\begin{tabular}{|l|}
\hline Pattern of collaboration pre and post intervention \\
\hline Types of collaborative practice agreements (CPA) \\
\hline Frequency of referral \\
\hline Physician perceptions CPA necessity \\
\hline Facilitators and barriers of collaboration (impact of physician \& pharmacist \\
\hline Expansion to other disease states \\
\hline Integration of model into usual primary care \\
\hline Physician receptiveness of pharmacist's recommendations pre and post \\
intervention \\
\hline Types of pharmacists recommendations accepted and denied \\
\hline Physician perception of pharmacist expertise pre and post intervention \\
\hline Outcomes of collaboration \\
\hline Perceived impact on patient-physician relationship \\
\hline Perceived impact on patient outcomes \\
\hline Observed patient perceptions of collaboration \\
\hline
\end{tabular}

Table 1: Summative evaluation domains.

\section{Data analysis}

A codebook for the summative evaluations was developed by the interviewer $(\mathrm{CP})$, and research team members (NB and $\mathrm{RF}$ ). The majority of the questions in the interview were either closed ended with a binary response such as "yes" or "no" or were open ended questions with free text responses. Microsoft Excel was used to record responses and themes were identified from the data. Qualitative data management software was not used for data analysis. Free text responses with similar themes were categorized together. For example, the physicians were asked what type of pharmacist recommendations they are more likely to accept. Responses such as dose adjustment, 
Page 3 of 7

medication initiation, medication adherence, and drug-drug interactions could all be collapsed under a category of "medication management".

Once the codebook was developed, the 3 members scored 1 physician response independently and scores were compared for reliability. After discussing the score from the first respondent, 4 more responses were scored independently by 2 reviewers and agreed upon through discussion and a consensus. A third reviewer adjudicated coding disagreements. After the first 5 responses were scored, the remaining physician responses were scored by 2 evaluators. A third individual reviewed the survey if a tiebreaker was needed. The codebook was updated after every group of 10 respondents were scored and discussed.

\section{Results}

The results of the main trial have been previously reported and are discussed here to demonstrate the acceptance of the intervention [18]. Briefly, the primary outcome of BP at 9 months was $43 \%$ at intervention sites and $34 \%$ at control sites (adjusted odds ratio, 1.57 [95\% confidence interval, 0.99-2.50]; $\mathrm{p}=0.059$ ). When the most recent 2014 BP guidelines were considered [21], BP control was achieved in $61 \%$ of intervention patients and $45 \%$ of usual care patients [(adjusted odds ratio for BP control, 2.03 [95\% CI 1.29-3.22], $\mathrm{p}=0.003$ ) [18]. The adjusted difference in mean systolic/diastolic BP between intervention and control groups at 9 months was $-6.1 /-2.9 \mathrm{~mm} \mathrm{Hg}(\mathrm{p}=0.002$ and $\mathrm{p}=0.005$, respectively). The primary outcome of the asthma arm in the usual care BP group was the sum of asthma-related emergency department (ED) visits and hospitalizations in three periods: 9 months before intervention, 9 months during, and 9 months after the intervention. Of the 126 patients in the asthma arm, the number of ED visits and/or hospitalizations decreased 30\% during the intervention $(p=0.052)$ and returned to preenrollment levels after the intervention was discontinued $(\mathrm{p}=0.83)$ [17].

A total of 63 physicians and 26 pharmacists completed the summative evaluation from 27 and 26 offices, respectively. At least 1 physician and pharmacist from each study site were successfully interviewed; however we were unable to obtain our goal of interviewing at least 3 physicians from each site. Responses to the telephone interview were compared to reveal four essential themes related to implementation of the PPCM model. According to physicians and pharmacists, successful implementation of this model requires these facilitators: (1) physician buy-in for pharmacists' provision of clinical services, (2) inclusion of the pharmacist on patient care teams and embedded within the office, (3) improvements to the workflow processes at clinical sites, and (4) development of reimbursement strategies to make pharmacist service provision viable. Another resounding theme that emerged from the interview responses was positive patient perspectives on team-based approaches in primary care settings. Table 2 displays the number of physicians' responses to key themes in the summative evaluation. The percentages represent the number of physicians with a correlated response out of physicians who responded to the associated question. The comments with highest frequency are listed.

\begin{tabular}{|l|l|l|}
\hline Barriers to Implementation & & $\begin{array}{l}\text { Number } \\
\text { (\%) }\end{array}$ \\
\hline Physician Buy-in & & $44(95 \%)$ \\
\hline & PCP buy-in & 29 \\
\hline
\end{tabular}

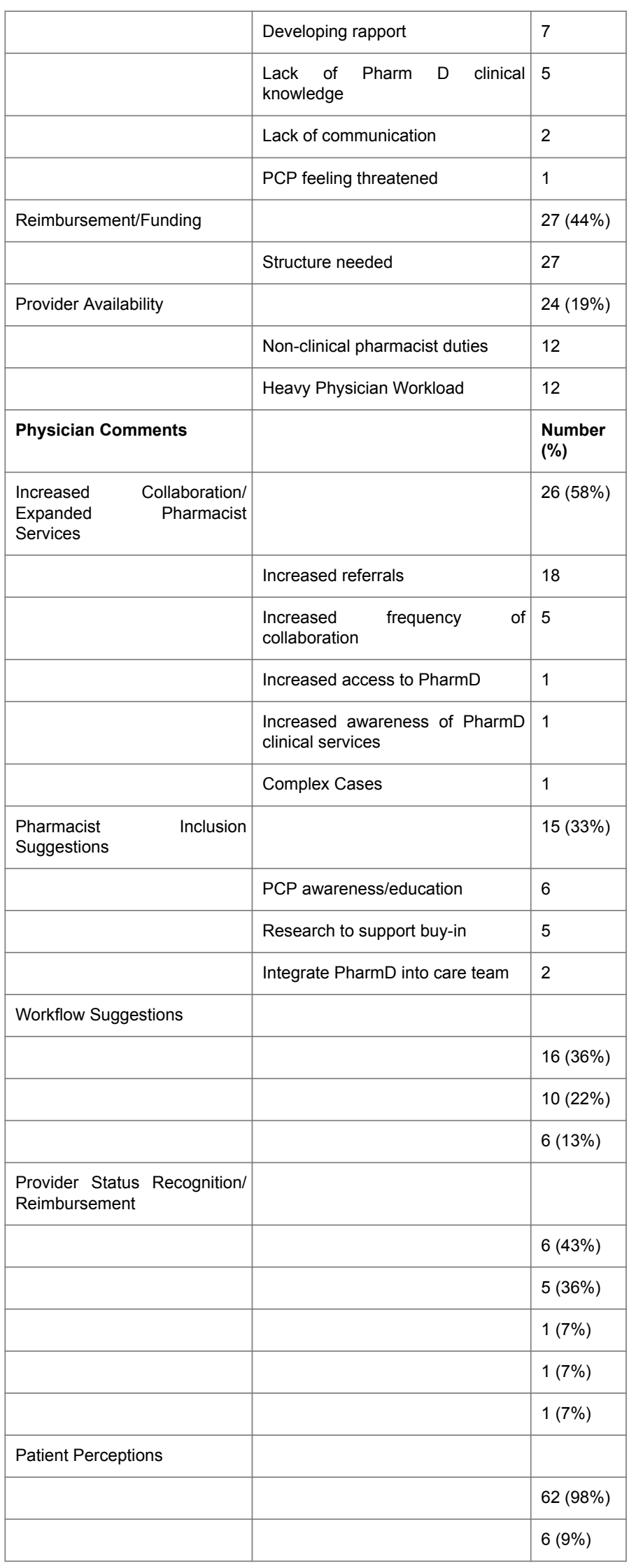

Table 2: Physician responses. 


\section{Physician buy-in for pharmacists' provision of clinical services}

Collaboration during the CAPTION trial resulted in expanded clinical pharmacy services for the management of chronic diseases. Those physicians who stated there was little or no change in the collaboration reported either a lack of collaboration related to pharmacist reimbursement limitations or a high level of collaboration prior to implementation of the study at their site. Regarding reimbursement limitations, physicians noted the need to "find ways to get reimbursed for pharmacist's time", and "identifying a reimbursement model" which would include "being able to sort out what portion of the reimbursement is applied to pharmacist's time". Pharmacists reported an overall increase in referrals for chronic disease management as a result of the intervention for the trial. The greatest level of collaboration and referral both before and after the intervention was for cardiovascular diseases including: diabetes mellitus, hypertension, dyslipidemia, anticoagulation, and congestive heart failure.

The majority of physicians believed collaboration with pharmacists is a necessary aspect of providing optimal care to patients. Pharmacists reported support from physicians for collaboration. One physician responded that pharmacist's involvement in chronic disease management "improves level of care and patient compliance". Physicians are confident in the expertise of pharmacists and their ability to manage chronic diseases overall. Despite the positive impact of PPCM during the CAPTION trial, physician buy-in for clinical pharmacist services remains the largest reported barrier to implementation of this model (Table 2). 7 physicians commented on rapport between physicians and pharmacists being a barrier and an area requiring improvement for successful implementation of the model. 5 physicians reported concerns regarding the clinical knowledge of pharmacists and identified this as a barrier to physician buy-in. The physicians who reported concerns with pharmacist expertise preferred team approaches to care rather than independent management of chronic diseases by pharmacists. Physicians commented on having a higher comfort level with pharmacists with whom they had a longstanding relationship.

Physicians reported high acceptance rates for pharmacist recommendations during the study and indicated that they were more likely to accept those related to cardiovascular diseases and medication therapy management, as well as those that were evidence-based. Most physicians noted high acceptance rates without specifying any particular type of recommendation and instead reported that they tended to "Accept All" recommendations from pharmacists. Physicians were less likely to accept recommendations if the pharmacist was not aware of relevant patient information pertaining to the recommendation.

\section{Pharmacist integration into patient care teams}

Most of the study offices had collaborative practice agreements (CPA) in place prior to the study intervention. Physicians reported reasons to implement a CPA that did not already exist as: improved patient care and outcomes, chronic conditions warranting collaboration, and heavy physician workload. The reasons physicians stated they may not create a CPA included: working well with pharmacists without a formal CPA, lack of pharmacist availability, reimbursement limitations for care provided by pharmacists, and feeling threatened by pharmacists in clinical roles performing functions that overlap with physician functions such as adjusting medications and ordering labs. A number of offices in the study were able to implement new CPAs for chronic disease management with some in process during the interview.

Physicians and pharmacists believe the model implemented in the CAPTION Trial could be expanded to disease states beyond BP and asthma (Table 3).

\begin{tabular}{|l|}
\hline Cardiovascular Disease \\
\hline Anticoagulation \\
\hline Congestive heart failure \\
\hline Coronary Artery disease \\
\hline Dyslipidemia \\
\hline Atrial fibrillation \\
\hline Diabetes \\
\hline Respiratory Disease \\
\hline COPD \\
\hline Mental Health \\
\hline Depression \\
\hline Anxiety \\
\hline Attention deficit disorder \\
\hline Chronic Pain \\
\hline Obesity \\
\hline Geriatrics \\
\hline
\end{tabular}

Table 3: Physician \& pharmacist recommended areas of expansion of services.

Physicians noted that their access to clinical pharmacists as well as awareness of clinical services provided by pharmacists increased as a result of the CAPTION Trial. Implementation of the PPCM model for collaborative care presents an opportunity to expand physician awareness and buy-in for the provisions of clinical services by pharmacists.

\section{Workflow processes}

Participants reported that collaboration between physicians and pharmacists generally increased as the physicians' workload became heavier. Physicians viewed pharmacist involvement in chronic disease state management as an opportunity to reduce physician workload. This observation is supported by CAPTION data analyzed by Polgreen et al which found a larger percentage of subjects in the control group had at least 1 physician visit in the 9 month study interval and had a higher median number of visits during the trial than the intervention group [19]. One physician noted that their willingness to collaborate could be influenced by the complexity of the patient, stating: "... when a case is complex it is worth the extra effort."

Pharmacist workload also appeared to impact collaboration. Issues related to reduced access to pharmacists including conflicts with other non-clinical responsibilities of the pharmacist such as: academic, 
administrative, and dispensing responsibilities were reported. Physicians noted that lack of availability of pharmacists was somewhat aided by the pharmacists' ability to delegate work to pharmacy students and residents. Clinic workflow issues that hinder collaboration included: room availability, running behind in clinic, and scheduling issues.

\section{Reimbursement for clinical services provided by pharmacists}

Most physicians believed pharmacists should be recognized as health care providers by Medicare and be able to bill for clinical services. One physician noted they were "surprised this was not already in place!" Previous reported data from the survey prior to the study determined how pharmacists received reimbursement for their services [20]. Pharmacist salaries were covered by patient revenue in $26.5 \%$ of practices. Approximately half (47.0\%) of the pharmacists charged for services rendered in "incident to" billing, and amounts charged in 2005 ranged from $<\$ 25,000$ (56.3\%), $\$ 25,000-\$ 99,999$ $(18.8 \%)$, and $>\$ 100,000(25.0 \%)$. Practice collections from patient care activities in 2005 ranged from $<\$ 25,000$ (71.4\%), $\$ 25,000-\$ 99,999$ (7.1\%), and $>\$ 100,000(21.4 \%)[20]$.

\section{Patient perspectives and patient relationship development}

Physicians and pharmacists alike reported a positive impact on patient-provider relationships resulting from PPCM including patients appreciating pharmacist input, viewing the collaboration as a team approach to their benefit, perceiving the collaboration as providing extra help or more expertise, and overall strengthening of patient relationships with their providers as well as improved quality of care. While 62 of the 63 responding physicians reported positive impact on patient-provider relationships, 6 of those physicians also identified negative patient impact (Table 2). Patient confusion regarding the pharmacists' role in clinic was reported by 3 individuals, 1 physician reported the potential for patients to be overwhelmed with an increased number of providers seen in one visit, 1 reported a negative impact associated with increased office visits for patients, and 1 reported negative impact related to increased patient cost.

All surveyed physicians responded that they believed a pharmacist's involvement in chronic disease management improved patient outcomes. Physicians and pharmacists believed that collaborating with one another was successful for improving patients' disease state control.

\section{Implementation of physician-pharmacist collaboration management}

When asked how physician-pharmacist collaboration can be integrated into usual primary care, both groups identified important areas that need to be addressed. First and foremost, successful integration of this model will require the pharmacist to be on site and available in the primary care setting. Secondly, pharmacists need to be included in the patient care team with time set aside for pharmacistprovided services. Equally important are the level of physicianpharmacist communication, implementation of an easy referral process, and development of reimbursement and/or funding opportunities for pharmacist-provided services to make integration into primary care successful.

Barriers to implementation of the collaborative model that were identified by participants included: insufficient physician buy-in primarily related to inadequate communication and rapport between physicians and pharmacists, limited availability of both providers and patients, and lack of reimbursement or funding for the provision of pharmacist clinical services. Facilitators of implementation of the collaborative model identified by both pharmacists and physicians included development of the physician-pharmacist relationship through good communication, inclusion of the pharmacist on the patient care team and within the workflow process, patient education regarding the pharmacists' role in the primary care setting, and physician education to improve awareness of pharmacist clinical services and the effectiveness of the model for improving patient outcomes. Some suggestions from physicians to facilitate collaboration included, "have physicians reflect on how pharmacists can add value to patient care and improve patient outcomes", "educating patients about the role of the pharmacist in the clinic", and "make interaction of pharmacist with patient and physician automatic and part of the formal process".

\section{Discussion}

The goal of this summative evaluation was to explore the perceptions of both physicians and pharmacists regarding their ability to impact and improve patient care through collaborative practice in the primary care setting. Overall, our results indicate physicians and pharmacists are open to collaborating in primary care settings and believe this collaboration will improve patient outcomes in the management of chronic diseases. Several physicians noted collaboration with a pharmacist was especially helpful for complex patients with multiple conditions and mediations. To be eligible for the study, patients had to have uncontrolled hypertension, however many of the patients in the study had multiple chronic disease states. Baseline data shows $47.4 \%, 47.3 \%$, and $54.0 \%$ of patients in the brief, sustained, and control arms had diabetes mellitus or kidney disease and $15.5 \%, 23.7 \%$, and $14.7 \%$ respectively were current smokers. At baseline, patients were taking an average of 2 antihypertensive medications and had an average of 2 comorbidities [18].

There are significant overlapping themes found in both the CAPTION qualitative assessment and Snyder et al [16]. Key similarities include physician buy-in as a facilitator, and barriers such as lack of pharmacist reimbursement of services, trust of pharmacists to perform clinical functions, lack of pharmacist time and resources, and existing informal collaborations causing decreased interest in obtaining formal collaborative agreements. Snyder et al identified leveraging academic partners as a facilitator however this was not a common theme identified through CAPTION pharmacist and physician interviews with only 1 study pharmacist suggested utilizing students as support to physician-pharmacist collaboration. In fact, 5 pharmacists identified academic responsibilities as a time barrier to collaborating.

The CAPTION Trial provided an opportunity to show the contributions that can be made by pharmacists when they are accessible as part of the healthcare team. Our summative evaluation also indicated that while much progress has been made, education of physicians, nurses, and other healthcare professionals regarding the value of this model and pharmacists as part of the healthcare team will be an ongoing necessity to move toward implementation of collaborative practice. McDonough and Doucette provide a description on the stages of developing and fostering positive working relationships with providers as well as provide guidance on advancing collaborations [22]. Results show access to pharmacist expertise and 
team approaches to provision of healthcare are a priority for healthcare providers in order to optimize patient care.

Our results also indicate a need for improved communication between physicians and pharmacists to ascertain roles and expectations for the services provided. Several respondents indicated concern about patients being confused regarding the role of pharmacists in the clinic. These 3 respondents were from clinics that either did not have a prior CPA or had an informal collaborative agreement. Patients from these clinics may not have had routine exposure to clinical pharmacists due to a less formal collaboration prior to the study which could explain confusion in the pharmacist's clinical role. Although access to pharmacists was identified as crucial, pharmacists are not always in the medical office or clinic full time. Most of the clinical sites in the study were family medicine residency training offices. Some of the pharmacists were regularly scheduled in the office; however, many pharmacists are also appointed as faculty members in colleges of pharmacy and therefore had academic responsibilities outside the office.

The biggest question yet to be addressed is how these clinical services provided by pharmacists and valued by physicians will be paid. Reimbursement and/or funding for the provision of clinical services by pharmacists is a primary barrier to implementation of collaboration into primary practice settings despite overwhelming perception by both pharmacists and physicians that this collaboration can and does improve patient care.

\section{Strengths and Limitations}

The CAPTION trial was conducted in 15 states throughout the country and thus responses represent physician and pharmacist perceptions from multiple regions throughout the United States. In this summative evaluation, interviewees were asked scripted questions by a single interviewer to ensure consistency of questioning. The questions were written specifically to address pharmacists' integration into primary care using the PPCM model. The line of questioning maintained opportunities for spontaneous comments from physicians and pharmacists.

Many of the offices involved in the CAPTION trial were residency training locations. This may have resulted in greater support for collaboration given potential history of experience with collaborative care and exposures to pharmacist clinical services prior to the intervention. Longstanding relationships between physicians and pharmacists at some sites may also have resulted in a higher rate of acceptance for recommendations as positive relationships may have already been developed. The interviewer was a pharmacist and thus respondents may have been inclined to respond positively, not wanting to report negative opinions.

\section{Future Research}

Our understanding of how physicians and pharmacists perceived collaborative practice in the primary care setting has been enhanced by this study. Our results suggest that collaboration between healthcare professionals is not only valued by physicians and pharmacists, but also that it is highly valued by the patients. Further research to determine best practices for implementation and reimbursement of pharmacist clinical services into primary care settings are needed to determine the efficiency and cost-effectiveness of this model. With healthcare costs consuming a consistently increasing proportion of our gross domestic product, we need to identify treatment models that provide the highest quality of care while minimizing overall costs.

\section{Conclusion}

The results of this summative evaluation indicate collaboration in primary care settings is perceived positively by providers as a means to improve the quality of patient care and patient outcomes and to strengthen patient-provider relationships. Physicians' desire to collaborate with pharmacists for the management of chronic disease states is promising but will require continual efforts to develop and maintain positive professional relationships as well as reimbursement strategies for clinical services provided by pharmacists.

\section{References}

1. Patient-Centered Primary Care Collaborative. History of the collaborative.

2. Agency for Healthcare Research and Quality. What is the PCMH? AHRQ's definition of the medical home.

3. Carter BL, Rogers M, Daly J, Zheng S, James PA (2009) The potency of team-based care interventions for hypertension: a meta-analysis. Arch Intern Med 169: 1748-1755.

4. Santschi V, Chiolero A, Burnand B, Colosimo AL, Paradis G (2011) Impact of pharmacist care in the management of cardiovascular disease risk factors: a systematic review and meta-analysis of randomized trials. Arch Intern Med 171: 1441-1453.

5. Santschi V, Chiolero A, Colosimo AL, Platt RW, Taffe P, et al. (2014) Improving blood pressure control through pharmacist interventions: a meta-analysis of randomized controlled trials. J Am Heart Assoc 3: e000718.

6. Nigro SC, Garwood CL, Berlie H, Irons B, Longyhore D, et al. (2014) Clinical pharmacists as key members of the patient-centered medical home: an opinion statement of the Ambulatory Care Practice and Research Network of the American College of Clinical Pharmacy. Pharmacotherapy 34: 96-108.

7. Carter BL, Bergus GR, Dawson JD, Farris KB, Doucette WR, et al. (2008) A cluster randomized trial to evaluate physician/pharmacist collaboration to improve blood pressure control. J Clin Hypertens 10: 260-271.

8. Carter BL, Ardery G, Dawson JD, James PA, Bergus GR, et al. (2009) Physician and pharmacist collaboration to improve blood pressure control. Arch Intern Med 169: 1996-2002.

9. Wentzlaff DM, Carter BL, Ardery G, Franciscus CL, Doucette WR, et al. (2011) Sustained blood pressure control following discontinuation of a pharmacist intervention. J Clin Hypertens (Greenwich) 13: 431-437.

10. Chen Z, Ernst ME, Ardery G, Xu Y, Carter BL (2013) Physicianpharmacist co-management and 24-hour blood pressure control. J Clin Hypertens (Greenwich) 15: 337-343.

11. Zillich AJ, Sutherland JM, Kumbera PA, Carter BL (2005) Hypertension Outcomes through blood pressure monitoring and evaluation by pharmacists (Home Study). J Gen Intern Med 20: 1091-1096.

12. Parker CP, Cunningham CL, Carter BL, Vander Weg MW, Richardson $\mathrm{KK}$, et al. (2014) A mixed-method approach to evaluate a pharmacist intervention for veterans with hypertension. J Clin Hypertens (Greenwich) 16: 133-140.

13. Carter BL, Barnette DJ, Chrischilles E, Mazzotti GJ, Asali ZJ (1997) Evaluation of hypertensive patients after care provided by community pharmacists in a rural setting. Pharmacotherapy 17: 1274-1285.

14. Patterson BJ, Solimeo SL, Stewart KR, Rosenthal GE, Kaboli PJ, et al. (2015) Perceptions of pharmacists' integration into patient-centered medical home teams. Res Social Adm Pharm 11: 85-95.

15. Oliveria SA, Lapuerta P, McCarthy BD, L'Italien GJ, Berlowitz DR, et al. (2002) Physician-related barriers to the effective management of uncontrolled hypertension. Arch Intern Med 162: 413-420. 
Citation: Finkelstein RJ, Blaine ND, Parker CP, Weg MWV, Carter BL (2016) Summative Evaluation of Physician-Pharmacist Collaboration Management. J Hypertens 5: 216. doi:10.4172/2167-1095.1000216

Page 7 of 7

16. Snyder ME, Earl TR, Gilchrist S, Greenberg M, Heisler H, et al. (2015) Collaborative drug therapy management: case studies of three community-based models of care. Prev Chronic Dis 12: E39.

17. Gums TH, Carter BL, Milavetz G, Buys L, Rosenkrans K, et al. (2014) Physician-pharmacist collaborative management of asthma in primary care. Pharmacotherapy 34: 1033-1042.

18. Carter BL, Coffey CS, Ardery G, Uribe L, Ecklund D, et al. (2015) A Cluster-randomized Trial of a Physician\Pharmacist Collaborative Model to Improve Blood Pressure Control. Circ Cardiovasc Qual Outcomes 8: 235-243.

19. Polgreen LA, Jayoung H, Carter BL, Ardery GP, Coffey CS, et al. (2015) Cost-Effectiveness of a Physician-Pharmacist Collaboration Intervention to Improve Blood Pressure Control. Am Heart Assoc 66: 1145-1151.
20. Dickerson LM, Kraus C, Kuo GM, Weber CA, Bazaldua OV, et al. (2007) Formation of a primary care pharmacist practice-based research network. Am J Health Syst Pharm 64: 2044-2049.

21. James PA, Oparil S, Carter BL, Cushman WC, Dennison-Himmelfarb C, et al. (2014) 2014 evidence-based guideline for the management of high blood pressure in adults: report from the panel members appointed to the Eighth Joint National Committee (JNC 8). JAMA. 311: 507-520.

22. McDonough RP, Doucette WR (2001) Developing collaborative working relationships between pharmacists and physicians. J Am Pharm Assoc 41: 682-692. 